\title{
Learning curve of image-guided video-assisted thoracoscopic surgery for small pulmonary nodules: A prospective analysis of 30 initial patients
}

\author{
Ming-Ju Hsieh, MD, ${ }^{a}$ Chih-Tsung Wen, MD, ${ }^{a}$ Hsin-Yueh Fang, MD, ${ }^{a}$ Yu-Wen Wen, PhD,,${ }^{a, b}$ \\ Chien-Cheng Lin, $\mathrm{ME}^{\mathrm{c}}$, and Yin-Kai Chao, $\mathrm{MD}^{\mathrm{a}}$
}

\begin{abstract}
Objectives: The use of image-guided video-assisted thoracoscopic surgery for simultaneous localization and removal of small solitary pulmonary nodules in a hybrid operation room using $\mathrm{C}$-arm cone-beam computed tomography is gaining momentum. We sought to assess the effect of the learning curve on procedural parameters and clinical outcomes of image-guided video-assisted thoracoscopic surgery for treating patients with small solitary pulmonary nodules.
\end{abstract}

Methods: Clinical variables and treatment outcomes of the 30 initial patients with solitary pulmonary nodules who were treated with image-guided video-assisted thoracoscopic surgery at Chang Gung Memorial Hospital (Taiwan) were prospectively analyzed. Two sequential groups (groups I and II, $\mathrm{n}=15$ each) were compared with regard to localization time, radiation doses, and success rates. We used the Pearson's correlation coefficient to investigate the association between the surgical experience and the procedural time.

Results: In the entire cohort, the median size of solitary pulmonary nodules on preoperative computed tomography images was $6 \mathrm{~mm}$ (interquartile range, 4.5-9 $\mathrm{mm}$ ), and their median distance from the pleural surface was $10 \mathrm{~mm}$ (interquartile range, $5-15 \mathrm{~mm}$ ). The median tumor depth-to-size ratio was 1.4 (interquartile range, 0.7-2.5). The clinical parameters were similar between the 2 groups. There was an inverse association between the surgical experience and the procedural time (Pearson's $r=-0.6873 ; P<.001$ ). A significant reduction in localization time (median, 24 vs 49 minutes, respectively; $P<.001$ ) and radiation exposure (median, 70.7 vs $224 \mathrm{mGy}$, respectively; $P<.001$ ) was noted in group II (late patients) compared with group I (early patients). Notably, the success rates in groups II and I were similar $(93.3 \%$ vs $86.7 \%$, respectively; $P=$. 876).

Conclusions: Our data demonstrate a significant learning curve for image-guided video-assisted thoracoscopic surgery in the treatment of solitary pulmonary nodules as evidenced by decreased localization time and radiation exposure occurring with increased surgical experience. (J Thorac Cardiovasc Surg 2018;155:1825-32)

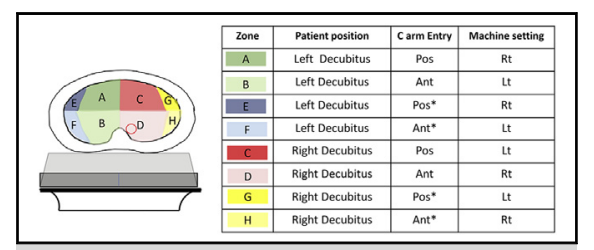

Schematic representation of optimal C-arm entry position according to lesion location.

\section{Central Message}

The reciprocal positioning of the patient, surgical table, and $\mathrm{C}$-arm is paramount to optimize the clinical outcomes of imageguided thoracoscopic surgery.

\section{Perspective}

Our data demonstrate a significant learning curve for iVATS as evidenced by decreased localization time and radiation exposure occurring with increased surgeon experience. The observed improvement is related to an increased confidence with regard to the reciprocal positioning of the patient, surgical table, and C-arm.

See Editorial Commentary page 1833.

\footnotetext{
From the a Department of Thoracic Surgery, Chang Gung Memorial Hospital, College of Medicine, Chang Gung University, Taoyuan, Taiwan; ${ }^{\mathrm{b}}$ Clinical Informatics and Medical Statistics Research Center, Chang Gung University, Taoyuan, Taiwan; and ${ }^{\mathrm{c}}$ Department of Advanced Therapy, Siemens Healthineers, Taipei, Taiwan.

This study was supported by Grants CMRPG3F1812 and CDRPG3B0011 13 from the Chang Gung Memorial Hospital, Taiwan.

Received for publication July 24, 2017; revisions received Oct 25, 2017; accepted for publication Nov 21, 2017; available ahead of print Jan 17, 2018.

Address for reprints: Yin-Kai Chao, MD, Division of Thoracic Surgery, Chang Gung Memorial Hospital-Linko, Chang Gung University, Taoyuan, Taiwan (E-mail: chaoyk@cgmh.org.tw).

$0022-5223 / \$ 36.00$

Copyright (C) 2017 by The American Association for Thoracic Surgery

https://doi.org/10.1016/j.jtcvs.2017.11.079
}

The use of low-dose computed tomography (CT) for lung cancer screening has led to an increased identification of solitary pulmonary nodules (SPNs). ${ }^{1}$ On identification of

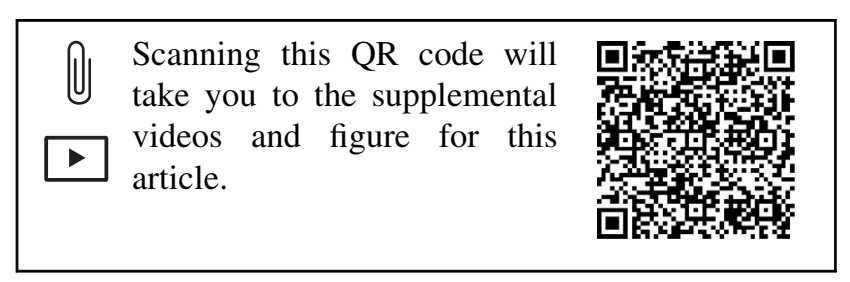




$$
\begin{aligned}
& \text { Abbreviations and Acronyms } \\
& \begin{aligned}
\text { CBCT } & =\text { cone-beam computed tomography } \\
\text { CT } & =\text { computed tomography } \\
\text { FOV } & =\text { field of visualization } \\
\text { GGN } & =\text { ground-glass nodule } \\
\text { IQR } & =\text { interquartile range } \\
\text { iVATS } & =\text { image-guided video-assisted } \\
& \text { thoracoscopic surgery } \\
\text { MDCT } & =\text { multiple detector computed tomography } \\
\text { OR } & =\text { operating room } \\
\text { SPN } & =\text { solitary pulmonary nodule } \\
\text { VATS } & =\text { video-assisted thoracoscopic surgery }
\end{aligned}
\end{aligned}
$$

an SPN, the subsequent clinical management should be based on the likelihood of the lesion to be malignant. Specifically, nodules that harbor cancerous potential should undergo biopsy or surgical removal. ${ }^{2}$ However, the removal of SPNs by video-assisted thoracoscopic surgery (VATS) may be challenging for thoracic surgeons, especially when the lesions are small or located deeply in the lung parenchyma. These SPNs are frequently thoracoscopically undetectable and less palpable through endoscopic instruments. $^{3-5}$

To increase the success rates of minimal invasive resection, several approaches (eg, use of hook wires, dyes, or microcoils) have been proposed to mark SPNs before VATS $^{6-9}$ To this aim, lesion localization is generally performed in a CT suite before transferring the patient to the operating room (OR). Notably, a smooth coordination between the CT suite and OR is paramount to ensure the success of the traditional 2-step approach, ultimately minimizing the risk of pneumothorax, hemothorax, or wire dislodgement associated with prolonged waiting times. ${ }^{10-12}$

The recent development of image-guided video-assisted thoracoscopic surgery (iVATS) has allowed the simultaneous localization and removal of SPNs in a hybrid OR using $\mathrm{C}$-arm cone-beam computed tomography (CBCT) equipped with a laser-guided navigation system. Despite a growing number of studies reporting the feasibility and safety of iVATS, no data are currently available on the learning curve for this technique. ${ }^{13-15}$ Therefore, this study was undertaken to assess the effect of the learning curve on procedural parameters and clinical outcomes of iVATS for treating patients with small SPNs. To this aim, clinical variables and treatment outcomes of the 30 initial patients with SPNs who were treated with iVATS at Chang Gung Memorial Hospital (Taiwan) were prospectively analyzed. All of the patients were operated by a single team of thoracic surgeons. Two sequential groups (group I and group II, $\mathrm{n}=15$ each) were compared with regard to localization time, radiation doses, and success rates.

\section{MATERIALS AND METHODS Study Sample}

We prospectively collected data from the first 30 patients with SPNs who were consecutively treated with iVATS in our institution over a 9-month period. All clinical and procedural data were entered and stored in a computerized database. For the purpose of comparison, we created 2 sequential patient groups $(\mathrm{n}=15$ each). Group I consisted of the first 15 patients, and group II consisted of the most recent 15 patients. The 2 groups were compared with regard to localization time, radiation doses, and success rates. The treatment protocol and data collection were granted ethical approval by our Institutional Review Board (CGMH-IRB 201600671A3). Written informed consent was obtained from all participants.

\section{Computed Tomography Indications for Nodule Localization and Selection of the Localization Method}

In the presence of solid nodules, localization was indicated for small subpleural $(<10 \mathrm{~mm})$ or deeply located nodules situated at a distance greater than $10 \mathrm{~mm}$ from the visceral pleura. Localization was also performed when subpleural cavitary lesions or ground-glass nodules (GGNs) were present. Preoperative CT-guided hook-wire localization was introduced in our hospital as of 2007 and has been adopted as the standard approach thereafter. ${ }^{7}$ Our iVATS program was commenced in 2016. When the hybrid OR (which is shared with the cardiovascular department) was available, patients in need of tumor localization underwent iVATS. If this was not the case, the traditional 2-stage approach was performed.

\section{Image-Guided Video-Assisted Thoracoscopic Surgery Workflow}

The entire iVATS procedure was performed in a hybrid OR equipped with CBCT (ARTIS zeego; Siemens Healthcare GmbH, Erlangen, Germany) and a Magnus surgical table (Maquet Medical Systems, Wayne, $\mathrm{NJ})$. After induction of general anesthesia and insertion of a double-lumen tube, both the patient's chest and the CBCT C-arm were wrapped in sterile covers. Under end-inspiratory breath-hold, we obtained an initial scan with a 6-second acquisition protocol (6s Dyna-CT Body) for surgical planning. The access path was laid out in the isotropic data set using the syngo Needle Guidance of a syngo X-Workplace (Siemens Healthcare GmbH). The needle path was initially defined by marking the entry and the target point of the needle and subsequently projected with a laser beam on the skin of the patient. Under end-inspiratory breath-hold, an 18-gauge marker needle was introduced into the thorax under 3-dimensional, laser-supported, fluoroscopic guidance. A laser-targeting cross was projected onto the patient's surface to visualize the needle entry point and angulation. Subsequently, the planned, virtual needle path was projected onto the live fluoroscopic image with the goal of correcting both needle orientation and positioning. The needle was subsequently introduced using a fluoroscopic "bull eye" approach until the tip was inside the projected target. Upon reaching the lesion, a localization wire (DuaLok; Bard Peripheral Vascular, Inc, Tempe, Ariz) was placed to localize the tumor. In case of superficial lesions, PBV dye $(0.3-0.5 \mathrm{~mL}$, patent blue V 2.5\%; Guerbet, Aulnay-sous-Bois, France) was injected to delineate the lesion. The accuracy of the needle path was verified by CBCT scans both throughout the procedure and on its completion. A VATS wedge resection was carried out under hook wire or dye guidance, and the resected lung lesion was submitted to frozen section examination. Lobectomy was the procedure of choice for all patients with a confirmed diagnosis of primary lung cancer. A sublobar resection was also considered the procedure of choice for patients with peripheral lung cancer of limited size $(<2 \mathrm{~cm})$ and adequate resection margins $(>2 \mathrm{~cm}$ or greater than tumor size). 
TABLE 1. General characteristics of the study participants

\begin{tabular}{|c|c|c|c|c|}
\hline Characteristics & Entire cohort $(n=30)$ & Group I $(n=15)$ & Group II $(n=15)$ & $P$ value \\
\hline Age, y; median (IQR) & $55.5(46-61)$ & $55(48.5-60)$ & $56(48-62)$ & .653 \\
\hline Sex & & & & .427 \\
\hline Male & $21(70 \%)$ & $12(80 \%)$ & $9(66.7 \%)$ & \\
\hline Female & $9(30 \%)$ & $3(20 \%)$ & $6(33.3 \%)$ & \\
\hline ASA physical status classification & & & & .651 \\
\hline I and II & $6(20 \%)$ & $4(27 \%)$ & $2(13 \%)$ & \\
\hline III & $24(80 \%)$ & $11(73 \%)$ & $13(87 \%)$ & \\
\hline CT findings & & & & .462 \\
\hline Solid nodule & $17(56.7 \%)$ & $10(67 \%)$ & $7(47 \%)$ & \\
\hline GGN & $13(43.3 \%)$ & $5(33 \%)$ & $8(53 \%)$ & \\
\hline Lesion size on CT, mm; median (IQR) & $6(4.6-8.9)$ & $6(4-8.5)$ & $8(5-8.8)$ & .461 \\
\hline Lesion location & & & & .466 \\
\hline Right-sided & $15(50 \%)$ & $6(40 \%)$ & $9(60 \%)$ & \\
\hline Left-sided & $15(50 \%)$ & $9(60 \%)$ & $6(40 \%)$ & \\
\hline Distance to the pleura, $\mathrm{mm}$; median (IQR) & $10(5-15)$ & $11.5(6-19.5)$ & $7(4-11)$ & .305 \\
\hline D-S ratio (IQR) & $1.4(0.7-2.5)$ & $1.9(1.0-2.8)$ & $1.2(0.5-1.7)$ & .161 \\
\hline
\end{tabular}

$I Q R$, Interquartile range; $A S A$, American Society of Anesthesiologists; $C T$, computed tomography; $G G N$, ground-glass nodule; $D-S$, depth-to-size.

\section{Definition of Outcomes and Statistical Analysis}

The localization time was calculated as the time elapsed from C-arm docking to the end of the localization procedure (ie, C-arm undocking). The total skin dose (expressed in mGy) was used as a measure of radiation exposure. Skin dose data were collected from the "Exam Protocol" of the ARTIS zeego instrument. Continuous data were expressed as medians and interquartile ranges (IQRs) and analyzed with the Mann-Whitney $U$ test. Categoric data were compared with the chi-square test or the Fisher exact test, as appropriate. We used the Pearson's correlation coefficient to investigate the association between the surgical experience and the procedural time. All statistical analyses were performed with the IBM SPSS 22.0 statistical package (IBM, Armonk, NY).

\section{RESULTS}

Table 1 summarizes the clinical characteristics of the entire study cohort, which was then divided into the 2 study groups. According to preoperative CT findings, 17 SPNs $(56.7 \%)$ were classified as solid lesions, and 13 (43.3\%) were GGNs. The median size of SPNs on preoperative CT images was $6 \mathrm{~mm}$ (IQR, 4.5-9 mm), whereas their median distance from the pleural surface was $10 \mathrm{~mm}$ (IQR, 5-15 mm). The median tumor depth-to-size ratio was 1.4 (IQR, 0.7-2.5). All of the clinical parameters were similar between the 2 groups.

TABLE 2. Technical details of the localization procedure

\begin{tabular}{|c|c|c|c|c|}
\hline Characteristics & Entire cohort $(\mathbf{n}=\mathbf{3 0})$ & Group I $(n=15)$ & Group II $(\mathbf{n}=15)$ & $P$ value \\
\hline Localization time, min; median (IQR) & $35(22.5-51.8)$ & $49(40-68)$ & $24(15-26)$ & $<.001$ \\
\hline $\begin{array}{l}\text { Patient position for localization } \\
\text { Lateral decubitus position } \\
\text { Others (supine or prone) }\end{array}$ & $\begin{array}{r}24(80 \%) \\
6(20 \%)\end{array}$ & $\begin{array}{l}9(60 \%) \\
6(40 \%)\end{array}$ & $\begin{array}{l}15(100 \%) \\
0(0 \%)\end{array}$ & .017 \\
\hline $\begin{array}{l}\text { Localization technique } \\
\text { Hook wire } \\
\text { Dye }\end{array}$ & $\begin{array}{l}17(56.7 \%) \\
13(43.3 \%)\end{array}$ & $\begin{array}{l}6(40 \%) \\
9(60 \%)\end{array}$ & $\begin{array}{r}11(73.3 \%) \\
4(26.7 \%)\end{array}$ & .139 \\
\hline Failure of localization & $3(10 \%)$ & $2(13.7 \%)$ & $1(6.7 \%)$ & 1.0 \\
\hline $\begin{array}{l}\text { No. of scans required for localization } \\
\leq 2 \\
>2\end{array}$ & $\begin{array}{r}21(70 \%) \\
9(30 \%)\end{array}$ & $\begin{array}{l}8(53.3 \%) \\
7(46.7 \%)\end{array}$ & $\begin{array}{r}13(86.7 \%) \\
2(13.3 \%)\end{array}$ & .109 \\
\hline $\begin{array}{l}\text { Radiation exposure } \\
\text { Computed tomography dose index, mGy; median (IQR) }\end{array}$ & $116.7(59.8-232.6)$ & $224.8(163.3-323.8)$ & $70.7(57.5-103.3)$ & $<.001$ \\
\hline $\begin{array}{l}\text { Final pathologic diagnosis } \\
\text { Primary lung cancer } \\
\text { Lung metastasis } \\
\text { Benign lesion }\end{array}$ & $\begin{array}{r}10(33.3 \%) \\
13(43.3 \%) \\
7(23.4 \%)\end{array}$ & $\begin{array}{l}4(26.7 \%) \\
7(46.6 \%) \\
4(26.7 \%)\end{array}$ & $\begin{array}{l}6(40 \%) \\
6(40 \%) \\
3(20 \%)\end{array}$ & 1.0 \\
\hline Postoperative LOS, d (IQR) & $4(3-5)$ & $4(3-4)$ & $4(3-5.5)$ & .381 \\
\hline
\end{tabular}

$I Q R$, Interquartile range; $L O S$, length of stay. 


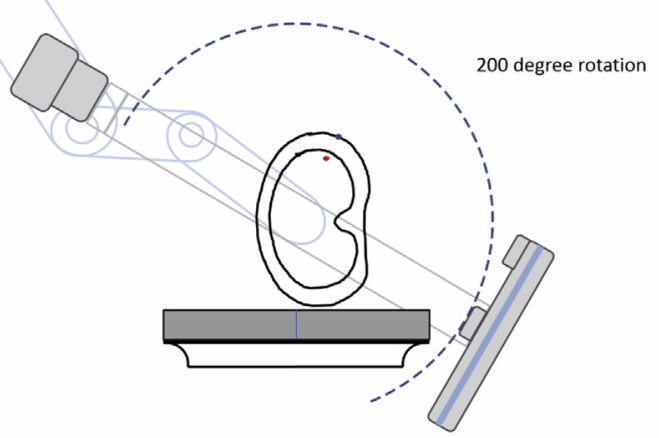

VIDEO 1. The acquisition of a Dyna-CT image requires a complete 200-degree rotation of the $\mathrm{C}$-arm around the surgical table. To include both the target lung lesion and the needle entry site into a unique CBCT FOV, the surgical table might be sporadically placed too low, ultimately leading to a collision with the C-arm. Video available at: http://www. jtcvsonline.org/article/S0022-5223(17)32817-9/fulltext.

Table 2 shows the technical details of the localization procedure. Twenty-four patients $(80 \%)$ were placed in the lateral decubitus position for localization, whereas the remaining 6 patients $(20 \%)$ were initially placed in supine or prone positions followed by repositioning to the decubitus position for surgery. All of the SPNs were identifiable on intraoperative CBCT images. Localization was performed by hook wire $(\mathrm{n}=17 ; 56.7 \%)$ or dye $(\mathrm{n}=13 ; 43.3 \%)$, being successful in 27 patients $(90 \%)$. The median total localization time was 35 minutes (IQR, 22-51.8 minutes), with the median radiation exposure being 116.7 mGy (IQR, 59.8-232.6 mGy). There was an inverse association between the surgical experience and the procedural time (Pearson's $r=-0.6873 ; P<.001$ ) (Figure E1). A significant reduction in localization time (median, 24 vs 49 minutes, respectively; $P<.001$ ) and
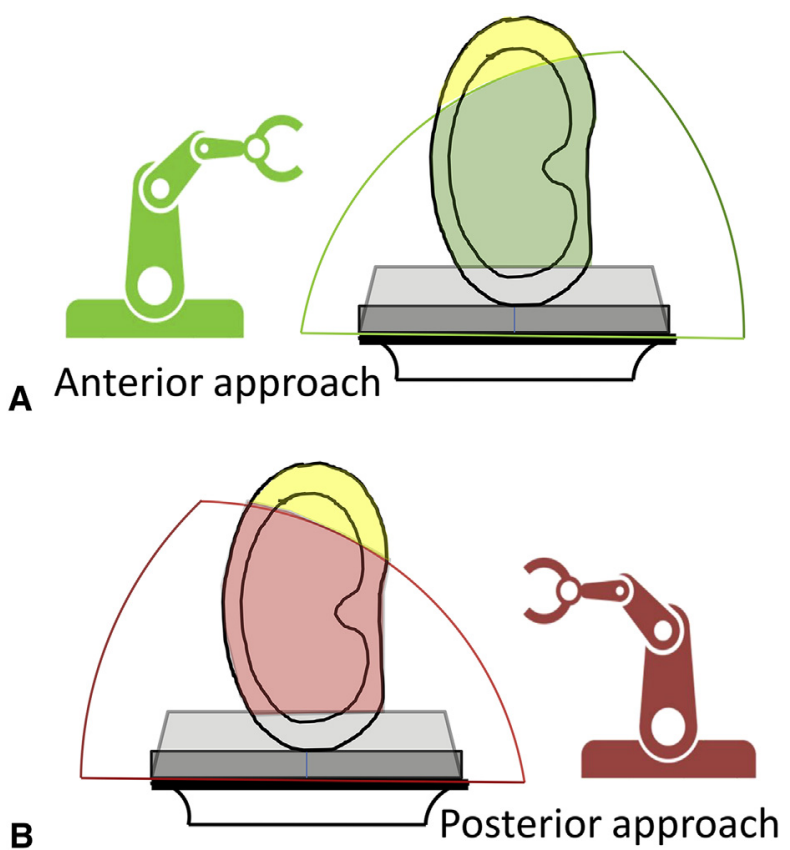

FIGURE 2. Schematic representation of the FOV of CBCT according to different $C$-arm entry positions. A, The area within the green polygon represents the range of FOV that can be obtained when a patient anterior C-arm entry position is selected. B, The area within the red polygon represents the range of FOV that can be obtained when a patient posterior $\mathrm{C}$-arm entry position is selected. The perspective for all figures is from the foot side to the head site of the surgical table as if the patient is lying in the right lateral decubitus position.

radiation exposure (median, 70.7 vs $224 \mathrm{mGy}$, respectively; $P<.001$ ) was noted in group II compared with group I. A successful lesion localization in the lateral decubitus position without any need of repositioning was significantly more common in group II than in group I ( $100 \%$ vs $60 \%$, respectively; $P<.001$ ).

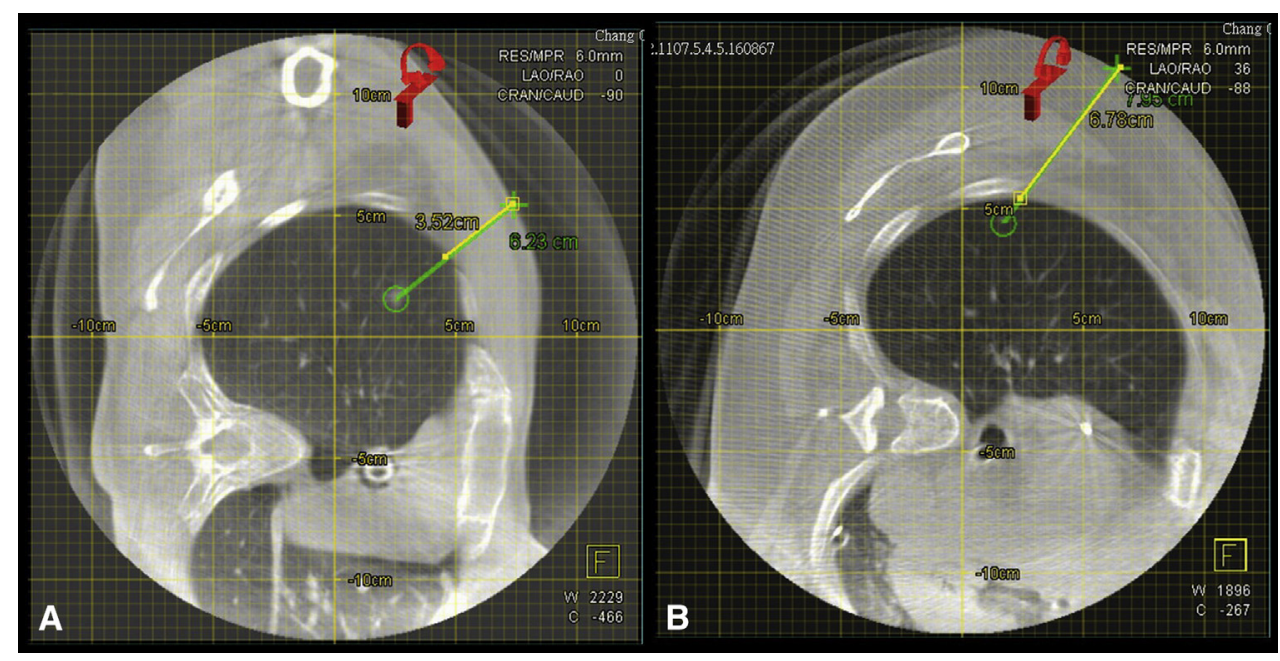

FIGURE 1. A, Representative image of an optimally designed field of visualization (FOV). Both the tumor (T) and the skin entry site (S) are visible in the same FOV and can successfully guide the needle path planning. B, Representative image of a poorly designed FOV. Only the tumor (T) site is visible, whereas the skin entry site is outside the FOV. A successful needle path planning cannot be achieved. 


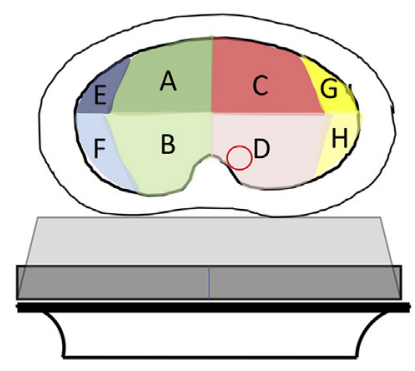

\begin{tabular}{|c|c|c|c|}
\hline Zone & Patient position & C arm Entry & Machine setting \\
\hline A & Left Decubitus & Pos & Rt \\
\hline B & Left Decubitus & Ant & Lt \\
\hline E & Left Decubitus & Pos* & Rt \\
\hline F & Left Decubitus & Ant* & Lt \\
\hline C & Right Decubitus & Pos & Lt \\
\hline D & Right Decubitus & Ant & Rt \\
\hline G & Right Decubitus & Pos* & Lt \\
\hline H & Right Decubitus & Ant* & Rt \\
\hline
\end{tabular}

FIGURE 3. Schematic representation of the optimized $C$-arm entry position in relation to patient position and lesion zone. The perspective for all figures is from the foot side to the head site of the surgical table as if the patient is lying in the right lateral decubitus position. *Patients were replaced to the semi-prone or semi-decubitus position.

There were 2 failures (due to pneumothorax induced by needle puncture) in group I. One failure caused by the inability to identify the dye injection site during iVATS occurred in group II. A small utility thoracotomy was created for exploration in all of the 3 failures. The neoplasm was identified under the guidance of a puncture hole on the lung surface and confirmed by digital palpation through the utility thoracotomy wound. All tumors were completely removed through a sublobar resection. The final results of pathology revealed the presence of cancer in 23 patients; margins were negative in all cases. All of the patients were successfully discharged from the hospital (median length of stay, 4 days; IQR, 3-5 days).

\section{DISCUSSION}

In the current study, we examined the results of our first 30 patients with SPNs who underwent iVATS by analyzing 2 groups of 15 consecutive patients. Our specific goal was to determine the effect of the learning curve on the development of proficiency with this increasingly popular technique. Our findings indicate that a definite procedure-related learning curve exists in iVATS, as shown by decreased localization time and radiation exposure in group II compared with group I. The significant decrease in localization time and radiation exposure observed in the more recent patient group highlights the importance of operator experience in this surgical setting. To our knowledge, this is the first study that specifically focused on the potential importance of the operator's experience as a critical factor for successful iVATS in patients with SPNs.

The localization time in our first 15 patients undergoing iVATS (group I; median, 49 minutes) was longer compared with previously published data concerning conventional localization performed in a CT room (median, $14 \sim 30 \mathrm{~min}){ }^{6,7,9,11}$ With increased procedural experience, the localization time was significantly reduced by more than $50 \%$ in the last 15 patients included in this study (group II; median, 24 minutes, $P<.001$ ). We believe that the observed reduction in localization time is related to (1) a better understanding of the complex arc geometry of the CBCT field of visualization (FOV) and (2) an improved appreciation of how an optimal FOV can be obtained by modifying the $\mathrm{C}$-arm spin location. As Video 1 shows, the acquisition of a Dyna-CT image requires a complete 200-degree rotation of the C-arm around the surgical table. To include both the target lung lesion and the needle entry site into a unique CBCT FOV, the surgical table might be sporadically placed too low, ultimately leading to a collision with the $\mathrm{C}$-arm. This concrete risk was especially evident when patients with a peripheral lung mass or a large chest cavity were placed in the lateral decubitus position (Figure 1, $A$, good FOV; Figure 1, $B$, poor FOV). In our initial 15 patients, $40 \%$ who were initially placed in the lateral decubitus position required a repositioning to the supine or prone position to complete the localization procedure (because of the occurrence of collision). We identified 4 helpful strategies to circumvent this issue. First, a careful selection of the optimal C-arm entry position according to the lesion location is required to obtain an optimal FOV. As shown in Figure 2, the FOV varied when the $\mathrm{C}$-arm entry position changed. A patient anterior C-arm entry position allowed a maximal FOV over the area corresponding to the posterior chest (Figure 2, A). Conversely, a patient posterior C-arm entry position allowed a maximal FOV over the anterior chest area (Figure 2, B). In light of these findings, a lesion located in an area over posterior chest had a higher likelihood to be fitted into the FOV obtained using a patient anterior entry position (making it the preferable choice). Figure 3 shows a schematic representation of the optimized C-arm entry position in relation to (1) the patient's position, (2) the machine setting, and (3) the lesion location. Figure 4 provides representative images obtained at different timing 


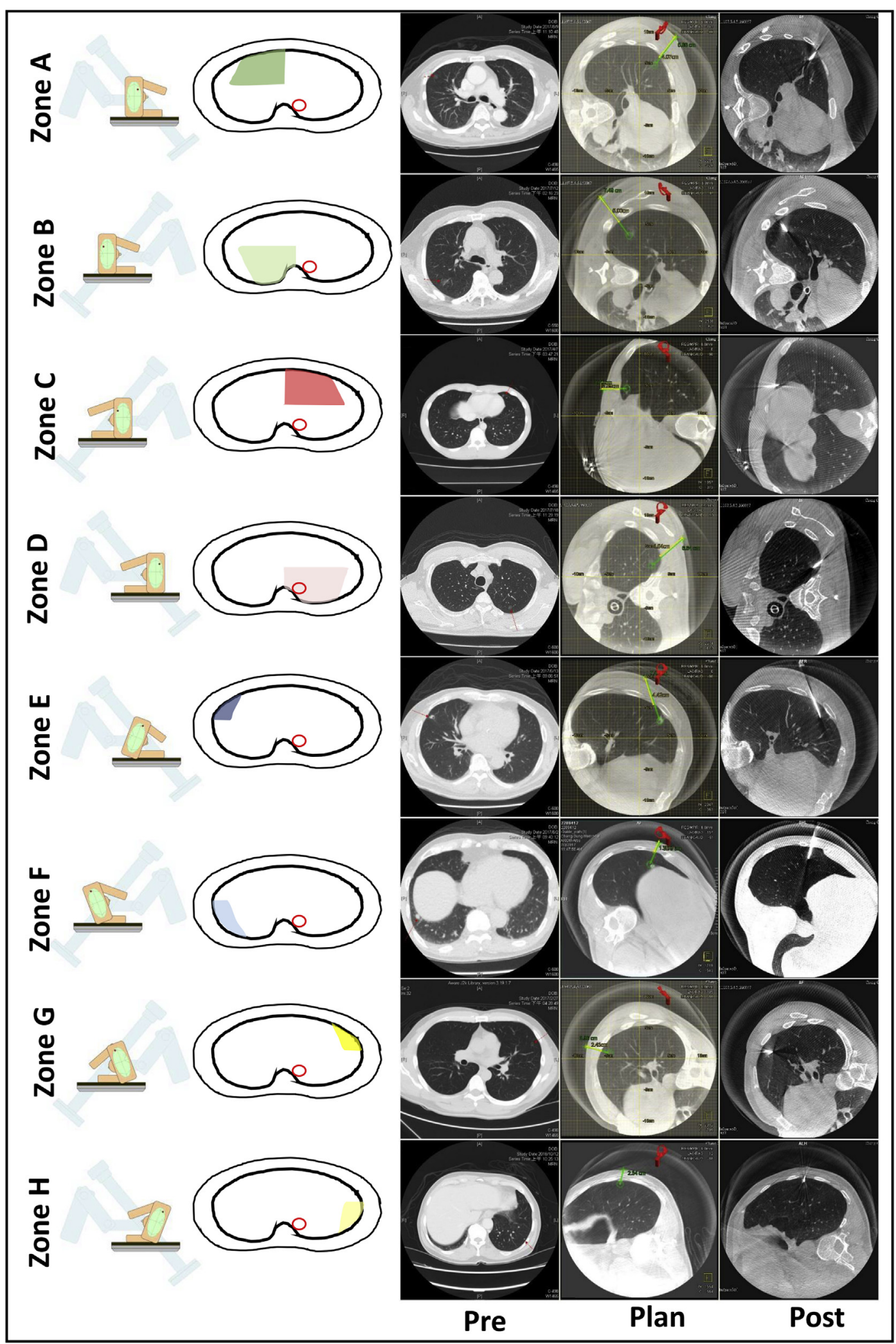

FIGURE 4. Representative images obtained at different time points. An MDCT image obtained before surgery (pre, left column), a CBCT image obtained during surgery (plan, middle column), and a CBCT image obtained after needle puncture (post, right column) are provided for lesions located at 8 different zones. 


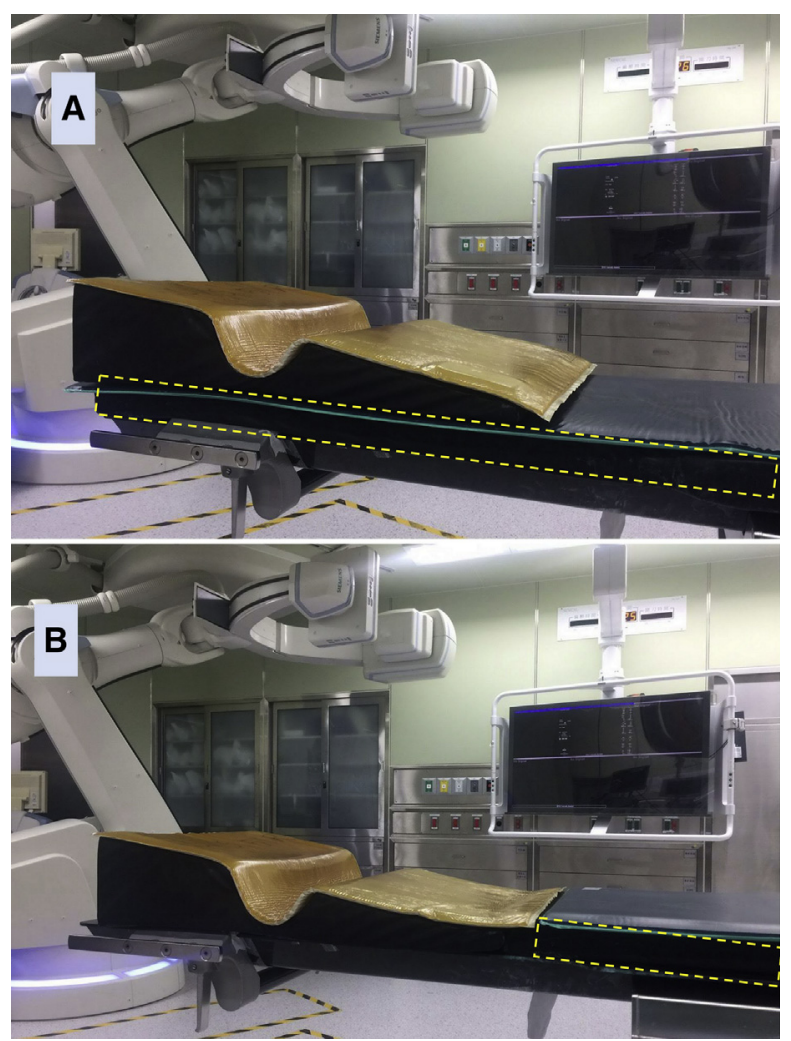

FIGURE 5. Hybrid OR equipped with CBCT (ARTIS zeego; Siemens Healthcare $\mathrm{GmbH}$, Erlangen, Germany) and a Magnus surgical table (Maquet Medical Systems, Wayne, NJ). The patient support pad (yellow dotted line), which was part of the surgical table, was removed to ensure a collision-free C-arm rotation. A, Before removal. B, After removal.

(before surgery, during the intraoperative planning, and after the needle puncture) of 8 lesions with different anatomic locations. Second, we tried to reduce the surgical table thickness (by $3-5 \mathrm{~cm}$ ) by acting on the patient's support pads and cushion (Figure 5). Third, the table should be moved from the midline toward the $\mathrm{C}$-arm side. A horizontal move of $6 \mathrm{~cm}$ would allow gaining an additional $3 \mathrm{~cm}$ in height, ultimately ensuring a collision-free C-arm rotation (Video 2). Fourth, we suggest rotating the patient lying in the decubitus position to a slightly semiprone or semidecubitus position when the 2 mentioned maneuvers are not sufficient (Video 2). The implementation of the 4 maneuvers (as we overcame the initial procedure-related learning curve) resulted in a statistically significant increase of successful SPNs localization in the lateral decubitus position over time (from $60 \%$ in group I to $100 \%$ in group II, $P=$. 017) (Table 2).

With increased surgical experience, we were also able to reduce the amount of radiation exposure. This achievement was not only caused by an optimized preoperative planning (which in turn lowered the number of unnecessary scans and fluoroscopy examinations) but was also related to a better

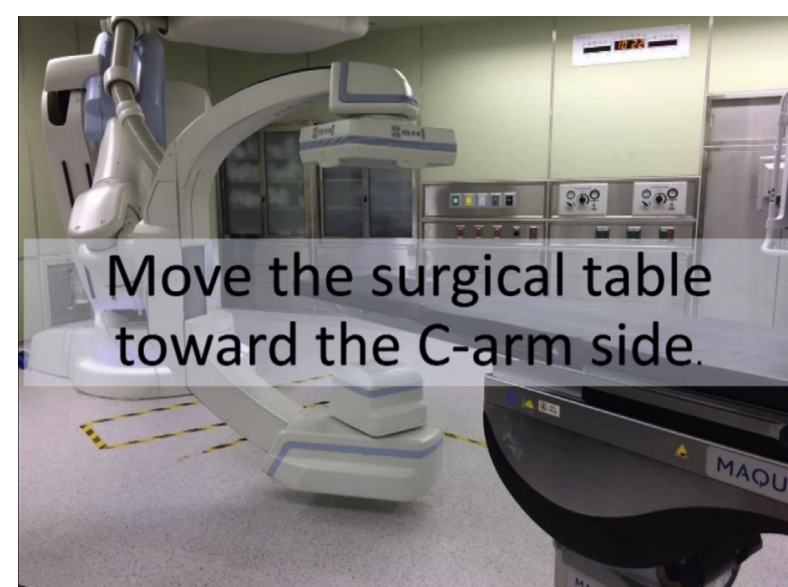

VIDEO 2. Strategies to avoid C-arm collision and maximize the feasibility of iVATS. First, the Magnus surgical table (Maquet Medical Systems, Wayne, NJ) should be moved toward the C-arm side. A horizontal move of $6 \mathrm{~cm}$ would allow gaining an additional $3 \mathrm{~cm}$ in height, ultimately ensuring a collision-free $\mathrm{C}$-arm rotation. Second, a patient lying in the decubitus position should be rotated to a slightly semiprone or semidecubitus position. Video available at: http://www.jtcvsonline.org/ article/S0022-5223(17)32817-9/fulltext.

reading of CBCT images and to improved skills in the use of the iGuide navigation system. In the presence of lesions other than GGNs, it was feasible to shift to a low-dose $5 \mathrm{~s}$ DR protocol (which allowed a $60 \%$ dose reduction compared with the standard 6s DCT protocol) without compromising the overall image quality. Remarkably, the protocol for acquiring Dyna-CT images was optimized over time. At least $3 \mathrm{CT}$ images (before, during, and after the procedure) were routinely acquired in early cases to ensure a reliable and accurate lesion localization. However, intraprocedural CBCT was omitted in most recent patients because of the high accuracy and reliability of the laser-targeting system (which allowed an additional reduction of radiation exposure).

In the current study, lung lesions were localized using a robotic C-arm CBCT. Besides C-arm CBCT, multiple detector computed tomography (MDCT) ${ }^{16}$ and mobile $\mathrm{O}$-arm $\mathrm{CBCT}^{17}$ have been used to localize pulmonary lesions in a hybrid OR. However, robotic $\mathrm{C}$-arm CBCT-guided tumor localization is superior to both MDCT and mobile O-arm CBCT in several instances. For example, the multi-axial, robotic technology-based system is highly flexible and allows localization and surgery to be completed on the same table (ie, without transferring the patient within the intra-hybrid OR). The achievement of this result is more difficult with the use of MDCT. Theoretically, mobile O-arm CBCT can offer a similar opportunity. However, the open gantry design of C-arm CBCT is more flexible during lesion targeting. Accordingly, we were able to complete the localization procedure with the patient lying in the decubitus position, 
with no subsequent modifications. Another point that merits consideration is the use of the iGuide navigation software. On determination of the needle path according to preprocedural CBCT data, the software allows marking the needle entry point through a laser cross. In addition, it offers a precise visualization of the virtual needle pathway when the target lesion is approached through fluoroscopy. Altogether, these characteristics allow avoiding repeated scans during needle targeting and highlight the superiority of C-arm CBCT over other imaging modalities for iVATS.

\section{Study Limitations}

Our findings need to be interpreted in the context of several limitations. First, the study cohort consisted of selected patients who had a single SPN requiring localization. The question as to whether iVATS can be safely applied to patients harboring 2 or more SPNs in need of localization remains open. Second, although we were able to show a decreased procedural time after the first 15 cases, our observations do not imply that the learning curve is invariably completed after treating 15 patients. The sample size of our study was small and did not allow us to calculate proficiency. Third, it is unclear whether the proposed single-stage approach can be more clinically successful or safe compared with the conventional 2-step technique. Further research with a larger number of patients is necessary to compare this novel technique with previously established localization methods in terms of diagnostic yields, complication rates, and costeffectiveness.

\section{CONCLUSIONS}

Our data demonstrate a significant learning curve for iVATS in the treatment of SPNs as evidenced by decreased localization time and radiation exposure occurring with increased surgeon experience. This approach can provide excellent treatment outcomes.

\section{Conflict of Interest Statement}

Authors have nothing to disclose with regard to commercial support.

\section{References}

1. National Lung Screening Trial Research Team, Church TR, Black WC, Aberle DR, Berg CD, Clingan KL, Duan F, et al. Results of initial low-dose computed tomographic screening for lung cancer. N Engl J Med. 2013;2013: 1980-91.
2. Naidich DP, Bankier AA, MacMahon H, Schaefer-Prokop CM, Pistolesi M, Goo JM, et al. Recommendations for the management of subsolid pulmonary nodules detected at CT: a statement from the Fleischner Society. Radiology. 2013;266:304-17.

3. Suzuki K, Nagai K, Yoshida J, Ohmatsu H, Takahashi K, Nishimura M, et al. Video-assisted thoracoscopic surgery for small indeterminate pulmonary nodules: indications for preoperative marking. Chest. 1999;115:563-8.

4. Saito H, Minamiya Y, Matsuzaki I, Tozawa K, Taguchi K, Nakagawa T, et al. Indication for preoperative localization of small peripheral pulmonary nodules in thoracoscopic surgery. J Thorac Cardiovasc Surg. 2002;124:1198-202.

5. Nakashima S, Watanabe A, Obama T, Yamada G, Takahashi H, Higami T. Need for preoperative computed tomography-guided localization in video-assisted thoracoscopic surgery pulmonary resections of metastatic pulmonary nodules. Ann Thorac Surg. 2010;89:212-8.

6. Lin M-W, Tseng Y-H, Lee Y-F, Hsieh M-S, Ko W-C, Chen J-Y, et al. Computed tomography-guided patent blue vital dye localization of pulmonary nodules in uniportal thoracoscopy. J Thorac Cardiovasc Surg. 2016;152:535-44.e532.

7. Chen Y-R, Yeow K-M, Lee J-Y, Su IH, Chu S-Y, Lee C-H, et al. CT-guided hook wire localization of subpleural lung lesions for video-assisted thoracoscopic surgery (VATS). J Formos Med Assoc. 2007;106:911-8.

8. Mayo JR, Clifton JC, Powell TI, English JC, Evans KG, Yee J, et al. Lung nodules: CT-guided placement of microcoils to direct video-assisted thoracoscopic surgical resection. Radiology. 2009;250:576-85.

9. Finley RJ, Mayo JR, Grant K, Clifton JC, English J, Leo J, et al. Preoperative computed tomography-guided microcoil localization of small peripheral pulmonary nodules: a prospective randomized controlled trial. J Thorac Cardiovasc Surg. 2015;149:26-32.

10. Watanabe KI, Nomori H, Ohtsuka T, Kaji M, Naruke T, Suemasu K. Usefulness and complications of computed tomography-guided lipiodol marking for fluoroscopy-assisted thoracoscopic resection of small pulmonary nodules: experience with 174 nodules. J Thorac Cardiovasc Surg. 2006;132:320-4.

11. Ichinose J, Kohno T, Fujimori S, Harano T, Suzuki S. Efficacy and complications of computed tomography-guided hook wire localization. Ann Thorac Surg. 2013; 96:1203-8.

12. Tomiyama N, Yasuhara Y, Nakajima Y, Adachi S, Arai Y, Kusumoto M, et al. CTguided needle biopsy of lung lesions: a survey of severe complication based on 9783 biopsies in Japan. Eur J Radiol. 2006;59:60-4.

13. Yang S-M, Ko W-C, Lin M-W, Hsu H-H, Chan C-Y, Wu I-H, et al. Image-guided thoracoscopic surgery with dye localization in a hybrid operating room. $J$ Thorac Dis. 2016;8:S681.

14. Kostrzewa M, Kara K, Rathmann N, Tsagogiorgas C, Henzler T, Schoenberg SO, et al. Computed tomography-assisted thoracoscopic surgery: a novel, innovative approach in patients with deep intrapulmonary lesions of unknown malignant status. Invest Radiol. 2017;52:374-80.

15. Gill RR, Zheng Y, Barlow JS, Jayender J, Girard EE, Hartigan PM, et al. Imageguided video assisted thoracoscopic surgery (iVATS)-phase I-II clinical trial. J Surg Oncol. 2015;112:18-25.

16. Ujiie H, Kato T, Hu HP, Patel P, Wada H, Fujino K, et al. A novel minimally invasive near-infrared thoracoscopic localization technique of small pulmonary nodules: a phase I feasibility trial. J Thorac Cardiovasc Surg. 2017;154:702-11.

17. Ohtaka K, Takahashi Y, Kaga K, Senmaru N, Kotani Y, Matsui Y. Video-assisted thoracoscopic surgery using mobile computed tomography: new method for locating of small lung nodules. J Cardiothorac Surg. 2014;9:110.

Key Words: ARTIS zeego, computed tomography, hybrid operating room, image-guided video-assisted thoracoscopic surgery, learning curve, small pulmonary nodules, video-assisted thoracoscopic surgery 


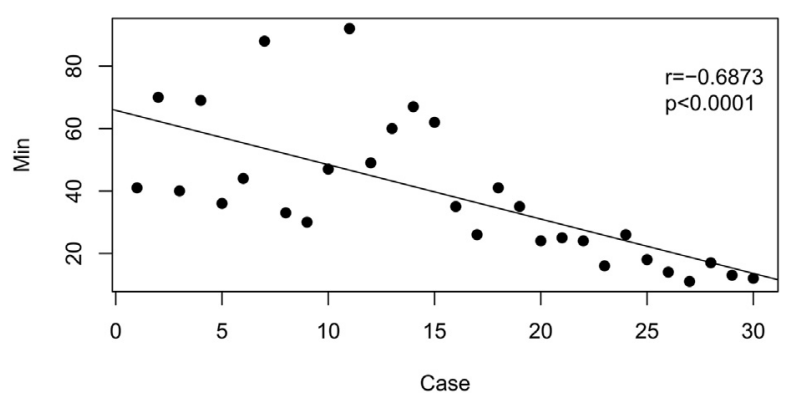

FIGURE E1. Representation of the learning curve in which the procedural time was plotted against the case number. 